\title{
Acquired Phrenic Nerve Injury
}

National Cancer Institute

\section{Source}

National Cancer Institute. Acquired Phrenic Nerve Injury. NCI Thesaurus. Code C103188.

Damage to the phrenic nerve that was not present at birth. 